\title{
Berezin Integration on General Fermionic Supermanifolds
}

\author{
Jeffrey M. Rabin` \\ The Enrico Fermi Institute of the University of Chicago, Chicago, IL 60637, USA
}

\begin{abstract}
Recent results on the global structure of supermanifolds are used to define a notion of Berezin integration on any purely fermionic Rogers supermanifold. This leads to an integration theory on a large class of supermanifolds having both bosonic and fermionic coordinates. The existence of global functions and forms on such supermanifolds is discussed, as is some elementary cohomology of supermanifolds.
\end{abstract}

\section{Introduction}

The Berezin integral as employed in superspace field theories is a formal operation with many of the properties of conventional integration. It is a linear map from polynomials in the anticommuting $\theta^{\alpha}$ coordinates to Grassmann numbers. Although it is thought of as a definite integral over the entire range of these coordinates, it has in fact no measure-theoretic interpretation. Thus, the corresponding indefinite integral is not defined, nor are definite integrals over finite regions [1-4].

Supermanifolds in the sense of A. Rogers [5] seem to be the most general objects on which the usual manipulations with superfields make sense. However, there is no general theory of integration on arbitrary Rogers supermanifolds. One might suspect that this is connected with the situation described above, and that no such integration theory will be forthcoming until the mathematical nature of the Berezin integral is better understood.

In this paper I will show that a notion of Berezin integration can be defined on any purely fermionic supermanifold (one having $\theta$ coordinates only). Unfortunately, this is not achieved via a deeper understanding of Berezin integration in flat superspace. Rather, it will be shown that an arbitrary fermionic supermanifold possesses so much structure that the usual formal definition of integration in flat superspace can be taken over. This result means that in principle action integrals for field theories on topologically nontrivial supermanifolds can be written down. This possibility is under investigation.

* Enrico Fermi Fellow. Research supported by the NSF (PHY 83- 01221) and DOE (DE-AC02-82ER-40073) 
The organization of the paper is as follows. In Sect. 2 I review the necessary results on the structure of supermanifolds [6-8], leading up to the crucial fact that any simply connected fermionic supermanifold immerses in flat superspace. Section 3 explores the implications of this fact for the algebras of global superfields and superforms. A simply connected fermionic supermanifold admits precisely as many global superforms as does flat superspace; it cannot be distinguished from flat superspace by the properties of these algebras. Section 4 contains the definition of the integral. The idea is to construct the universal covering space of a fermionic supermanifold, lifting the algebra of superforms to this covering space. Since the covering space is simply connected, it immerses in flat superspace, and the usual definition of integration can be pulled back using the immersion. Section 5 contains conclusions and implications for integration theory on general supermanifolds. An Appendix contains some remarks on the $G^{\infty}$ cohomology of supermanifolds and its relation to de Rham cohomology.

\section{The Structure of Fermionic Supermanifolds}

A supermanifold is built over a Grassmann algebra in much the same way that a complex manifold is built over the algebra of complex numbers. Thus, let $v_{1}$, $v_{2}, \ldots, v_{L}$ be generators of a Grassmann algebra $B_{L}$, satisfying $v_{i} v_{j}=-v_{j} v_{i}$. An arbitrary even element of this algebra can be written

$$
x=x_{0}+x_{i j} v_{i} v_{j}+x_{i j k l} v_{i} v_{j} v_{k} v_{l}+\cdots \equiv x_{\Gamma} v_{\Gamma},
$$

and an odd element appears as

$$
\theta=\theta_{i} v_{i}+\theta_{i j k} v_{i} v_{j} v_{k}+\cdots \equiv \theta_{\Sigma} v_{\Sigma}
$$

with the convention that only terms with subscripts in increasing order appear.

Flat superspace of dimension $(m, n)$, denoted $B_{L}^{m, n}$, is simply Euclidean space of dimension $2^{L-1}(m+n)$, with real coordinates denoted $x_{\Gamma}^{\mu}, \theta_{\Sigma}^{\alpha}$. Here $\mu$ runs from 1 to $m, \alpha$ runs from 1 to $n$, and $\Gamma$ and $\Sigma$ denote increasing sequences of indices as above. Grassmann coordinates $x^{\mu}, \theta^{\alpha}$ are built from the real coordinates as in Eqs. (2.1) and (2.2). On flat superspace there is a special class of $B_{L}$-valued functions called $G^{\infty}$ functions, or superfields. They have polynomial dependence on the odd coordinates,

$$
F(x, \theta)=f_{0}(x)+f_{\alpha}(x) \theta^{\alpha}+f_{\alpha \beta}(x) \theta^{\alpha} \theta^{\beta}+\cdots .
$$

Furthermore, the coefficient functions in this expansion are required to have Taylor expansions of the form,

$$
f(x)=f\left(x_{0}\right)+\partial_{\mu} f\left(x_{0}\right) s\left(x^{\mu}\right)+\frac{1}{2} \partial_{\mu} \partial_{v} f\left(x_{0}\right) s\left(x^{\mu}\right) s\left(x^{v}\right)+\cdots,
$$

with $s(x) \equiv x-x_{0}$. Note that the expansion (2.3) terminates provided only that $n$ is finite, while (2.4) terminates if $L$ is finite.

A supermanifold of dimension $(m, n)$ is a real manifold locally diffeomorphic to $B_{L}^{m, n}$ and having $G^{\infty}$ transition functions. One can show that any supermanifold has a foliation, called the soul foliation, by surfaces on which the "body coordinates" $x_{0}^{\mu}$ in any chart are constant. It is critically important that each simply connected leaf of 
this foliation can be immersed in the flat Euclidean space $R^{d}, d=2^{L-1}(m+n)-m$ [7].

I now specialize to a supermanifold $M$ of dimension $(0, n)$ and explain the construction of this immersion. The soul foliation has only a single leaf, which is all of $M$. A $G^{\infty}$ function on $M$ is a polynomial in the $\theta$ coordinates, thus also a polynomial in the real coordinates $\theta_{\Sigma}^{\alpha}$. Now a polynomial is completely determined by its values in any open set. This means that each transition function of $M$, initially defined only in an open subset of $B_{L}^{0, n}$, extends uniquely to a $G^{\infty}$ coordinate transformation on all of $B_{L}^{0, n}$. Now take a good covering of $M$ by charts $U_{j}$ and choose an initial chart $U_{0}$. (A good covering simply means that the intersection of any two charts should be contractible to a point.) It is mapped into $B_{L}^{0, n}$ in a $G^{\infty}$ manner by its coordinate map $\phi_{0}: U_{0} \rightarrow B_{L}^{0, n}$. The idea is to extend this to a map of all of $M$ into $B_{L}^{0, n}$. Take a chart $U_{1}$ which overlaps $U_{0}$. There will be a coordinate map $\phi_{1}: U_{1} \rightarrow B_{L}^{0, n}$ and a $G^{\infty}$ coordinate transformation $g=\phi_{0} \phi_{1}^{-1}$ on the overlap region. But $g$ extends uniquely to a coordinate transformation on all of $B_{L}^{0, n}$. This means that it is possible to change the coordinates in $U_{1}$ to agree with those of $U_{0}$ in the overlap region, and thereby extend the coordinate map $\phi_{0}$ to map all of $U_{0} \cup U_{1}$ into $B_{L}^{0, n}$. This extended map is not guaranteed to be one-to-one, although it is one-toone when restricted to either chart (some points in $U_{1}$ but not in $U_{0}$ may have been assigned the same coordinates as points in $U_{0}$ ). Therefore it is an immersion, not an embedding. This procedure can be continued until $\phi_{0}$ has been extended to all the charts $U_{j}$. Nothing can go wrong unless there is a topologically nontrivial closed loop in $M$. Going around such a curve while changing coordinates in the charts encountered can lead to an inconsistency upon returning to the initial chart. Thus, the result is that any simply connected supermanifold of dimension $(0, n)$ can be immersed in $B_{L}^{0, n}$ by a $G^{\infty}$ map. This immersion is unique once the coordinate map $\phi_{0}$ of the initial chart is chosen. In other words, it is unique up to an overall $G^{\infty}$ coordinate transformation on $B_{L}^{0, n}$.

Obviously no such result holds if $M$ is not simply connected. However, the universal covering space of $M$ can always be given a supermanifold structure and is simply connected.

\section{Superfields on Fermionic Supermanifolds}

I now discuss some properties of the algebra of superfields on a fermionic supermanifold $M$. Once again the basic fact is that superfields are polynomial functions of the coordinates. They are therefore uniquely determined by their values in an open set. This means that if a superfield is given locally, in a particular coordinate chart, it has at most one global extension. In flat superspace, of course, any local superfield has a global extension, but this need not be true when nontrivial topology is present. In general, then, the algebra of global superfields on $M$ will be smaller than the corresponding algebra on $B_{L}^{0, n}$. As an extreme example, it was shown in ref. [6] that if $M$ is compact the only global superfields are constants. The remarkable fact, however, is that if $M$ is simply connected it cannot be distinguished from $B_{L}^{0, n}$ by means of its algebra of superfields.

Let $M$ be an arbitrary fermionic supermanifold and $\tilde{M}$ its universal cover, which 
is also a supermanifold. Let $F$ be a superfield on $\tilde{M}$, initially defined only locally within some chart $U_{0}$. Via the coordinate map $\phi_{0}$ of $U_{0}, F$ becomes a superfield defined in a small region of $B_{L}^{0, n}$. But in $B_{L}^{0, n}$ any local superfield has a global extension. This global superfield can be pulled back to $\tilde{M}$ using the immersion which extends $\phi_{0}$, giving a global superfield on $\tilde{M}$.

Thus, on $\tilde{M}$ any local superfield has a global extension. This is true simply because $\pi_{1}(\tilde{M})=0$, and is independent of any other topology $\tilde{M}$ may have. The same argument holds for $G^{\infty}$ differential forms and tensors on $\tilde{M}$, objects which are locally polynomials in $d \theta^{\alpha}$ and $\partial / \partial \theta^{\alpha}$ with $G^{\infty}$ functions as coefficients. All such local objects have unique global extensions on $\tilde{M}$.

Even the $G^{\infty}$ cohomology of $\tilde{M}$ is the same as that of $B_{L}^{0, n}$. This simply means that any $G^{\infty}$ form obeying $d \omega=0$ is exact: $\omega=d \psi$ for some global $G^{\infty}$ form $\psi$. The proof is simple: certainly $\omega=d \psi$ locally, but then $\psi$ has a unique global extension. Since $\omega$ and $d \psi$ agree locally and have unique global extensions, they are equal globally and $\omega$ is exact. Some further basic properties of $G^{\infty}$ cohomology, including its relation to ordinary de Rham cohomology, can be found in the Appendix.

Thus, $\tilde{M}$ is indistinguishable from $B_{L}^{0, n}$ by the properties of its algebra of $G^{\infty}$ functions and forms. Now $M$ is the quotient space of $\tilde{M}$ by the group of covering transformations, which is also $\pi_{1}(M)$. A global $G^{\infty}$ form of $\tilde{M}$ gives rise to one on $M$ precisely when it is invariant under this group. The algebra of forms on $M$ is therefore typically smaller, and its cohomology may be nontrivial. However, the relation between $M$ and $\tilde{M}$ is strong enough to define integration on $M$ once it has been defined on $\tilde{M}$.

\section{Berezin Integration}

First of all, one must decide what sorts of global objects can be integrated over a fermionic supermanifold. Although the integrand of a Berezin integral is normally written locally as $f(\theta) d^{n} \theta$, the coordinate transformation law assigned to the expression $d^{n} \theta$ shows that it is not a product of coordinate differentials. This transformation law, in fact, is appropriate to the tensor field written locally as

$$
\frac{\partial}{\partial \theta^{1}} \frac{\partial}{\partial \theta^{2}} \cdots \frac{\partial}{\partial \theta^{n}},
$$

and I will adopt the notation $\partial^{n} \theta$ rather than $d^{n} \theta$ for this object. The objects which are integrated are therefore $n^{\text {th }}$ rank tensor fields which live in the $n$-fold product of the tangent bundle rather than the cotangent bundle $[2,9]$.

This creates a certain technical problem because of the fact that the object $\partial / \partial \theta^{\alpha}$ is not even locally a vector field on a supermanifold whose Grassmann algebra $B_{L}$ is finite dimensional $[10,11]$. This is because it does not obey the Leibniz rule for the derivative of a product. To see this, consider

$$
\frac{\partial}{\partial \theta^{1}}\left(v_{1} v_{2} \cdots v_{L} \theta^{1}\right)
$$

If the Leibniz rule were valid, this derivative would have to be $v_{1} v_{2} \cdots v_{L}$, but this is 
impossible because the function being differentiated is identically zero! Thus, the volume element (4.1) is not an element of the $n$-fold product of the tangent bundle when $L$ is finite.

The simplest response to this difficulty is to consider only supermanifolds constructed over $B_{\infty}$, which completely avoids the problem. Indeed, this is the case of interest in physics, since it is always assumed that the Grassmann algebra contains as many algebraically independent elements as may be needed, not merely $L$ such elements. All the arguments in this paper are valid in this infinite dimensional context. Nevertheless, one would like to define integration for the finite dimensional supermanifolds as well.

Although $\partial / \partial \theta^{\alpha}$ is not a vector field when $L$ is finite, the operator $f(\theta)\left(\partial / \partial \theta^{\alpha}\right)$ is a vector field when $f(\theta)$ is nilpotent (has no real part). In particular the expression

$$
\theta^{1} \cdots \theta^{n} \partial^{n} \theta
$$

whose Berezin integral in flat superspace is unity, is well-defined. The formal integrands which are not well-defined all integrate to zero according to the usual rules. This means that all the nonzero integrals of the usual formal approach can be obtained from well-defined integrands. This has the amusing consequence that although tensors can be integrated on finite-dimensional supermanifolds, functions strictly speaking cannot, because the volume element alone is not well-defined.

Now I will define the Berezin integral over an arbitrary fermionic supermanifold $M$. An $n^{\text {th }}$ rank tensor on $M$ can be viewed as an $n^{\text {th }}$ rank tensor on $\tilde{M}$ which is invariant under covering transformations. Its integral is defined to be equal to the corresponding integral on $\tilde{M}$. It remains to define integration on simply-connected supermanifolds like $\tilde{M}$. A tensor $\omega$ on $\tilde{M}$ is the pullback of a tensor $\hat{\omega}$ on $B_{L}^{0, n}$ via an immersion. Simply define

$$
\int_{\widetilde{M}} \omega=\int_{B_{L}^{0, n}} \hat{\omega}
$$

where the integral on the right is given by the usual Berezin integration rules. The definition seems ambiguous because $\hat{\omega}$ depends on which of the many possible immersions is used. However, different immersions differ only by overall $G^{\infty}$ coordinate transformations of $B_{L}^{0, n}$, and the Berezin integral is invariant under such transformations. Therefore the definition (4.2) makes sense.

Although this discussion may seem abstract, the practical rule for Berezin integration on arbitrary supermanifolds is extremely simple. To integrate a suitable tensor field, write it out in the coordinates of any chart. Then simply compute the integral by the same formal rules one would use in flat superspace. The point of the abstract discussion above is simply to prove that the result is independent of the choice of chart. The immersion one is implicitly using here is the one which extends the coordinate map of the chosen chart.

One consequence of this definition underlines the fact that the Berezin integral is not a measure-theoretic object, being completely insensitive to the "volume" of $M$. 
Namely, if $N$ is an open subset of $M$, and $\omega$ is an integrable tensor field on $M$, then

$$
\int_{N} \omega=\int_{M} \omega
$$

\section{Conclusions}

I have shown that the extremely rigid structure of fermionic supermanifolds allows a notion of Berezin integration to be defined. The central principle is that a $G^{\infty}$ function is uniquely determined by its values in an open set. This implies that all global objects are uniquely determined once given in a particular chart. The only topological property which affects the global existence of locally defined objects is the first homotopy group. This reduces the case of an arbitrary $M$ to the simplyconnecting covering space $\tilde{M}$.

Of course, supermanifolds of physical interest have commuting as well as anticommuting coordinates. Integration on purely bosonic supermanifolds (commuting coordinates only) is now well understood $[3,4,12]$. One integrates a differential form of highest degree over an embedded body submanifold, namely a submanifold which is transverse to the leaves of the foliation described in Sect. 2. This integral is invariant under deformations of the body which leave its boundary fixed. By combining this with the results of the present work one obtains a complete integration theory on supermanifolds of the form $B \times F$ with $B$ purely bosonic and $F$ purely fermionic. More generally the supermanifold need only have the structure $B \times F$ locally, as will now be discussed.

The objects which can be integrated on a general supermanifold of dimension $(m, n)$ are the "global integral forms" [13]. These are forms which appear as

$$
F(x, \theta) d^{m} x \partial^{n} \theta
$$

in some set of charts which covers the supermanifold. Rogers has shown that the transition functions relating charts in this set necessarily have the property that $\partial x^{\prime \mu} / \partial \theta^{\alpha}=0$ : the even coordinates of one chart depend only on the even coordinates of the other chart. This means that when global integral forms exist the supermanifold has a foliation by surfaces of constant $x$, because when charts overlap a surface of constant $x$ will also be a surface of constant $x^{\prime}$. Only the $\theta$ coordinates vary along these surfaces, so this foliation might be termed the $\theta$ foliation, to be distinguished from the soul foliation. Suppose now that the supermanifold is actually a fiber bundle with the leaves of the $\theta$ foliation as fibers and a base space parametrized by the $x$ coordinates. The results of this paper suffice to provide an integration theory over all such supermanifolds, first using Berezin integration to integrate over the fibers and then integrating over the body.

What prevents the development of an integration theory for arbitrary supermanifolds using the ideas of this paper? For general supermanifolds one knows that each leaf of the $\theta$ foliation has a covering space which immerses in $B_{L}^{0, n}$, so that an integral over each leaf can be defined using the ideas presented here. One would expect Berezin integration over the leaves to produce a form on the space of leaves which could then be integrated over the even coordinates. In general, however, the space of leaves of the $\theta$ foliation is not a smooth manifold, so this idea cannot be 
implemented. There is probably no sensible notion of integration over a supermanifold whose $\theta$ foliation is badly behaved.

\section{Appendix}

Here I discuss some basic properties of the $G^{\infty}$ cohomology of a supermanifold, including the connection with de Rham cohomology which establishes that $G^{\infty}$ cohomology really does have topological significance. Supermanifolds having both even and odd coordinates will be considered.

Given a supermanifold $M$ one can consider several different complexes of differential.forms. First, since $M$ is an ordinary real manifold, there is the usual de Rham complex of real-valued differential forms. These locally appear as polynomials in the coordinate differentials $d x_{\Gamma}^{\mu}, d \theta_{\Sigma}^{\alpha}$ having smooth real-valued functions as coefficients. The differential operator is

$$
d=d z_{\Gamma}^{A} \frac{\partial}{\partial z_{\Gamma}^{A}},
$$

where $z_{\Gamma}^{A}$ runs over the real coordinates. The cohomology of this complex, namely the algebra of closed forms modulo exact forms, will be denoted $H_{D R}(M)$. Second, there is the complex of $B_{L}$-valued differential forms, defined exactly as above except with smooth coefficient functions valued in the Grassmann algebra $B_{L}$. This defines the cohomology $H_{D R}\left(M ; B_{L}\right)$. Finally, there is the complex of $G^{\infty}$ differential forms which actually reflects the supermanifold structure of $M$. These forms locally appear as polynomials in $d x^{\mu}$ and $d \theta^{\alpha}$ having $G^{\infty}$ coefficient functions. (When $M$ is finitedimensional, $d \theta^{\alpha}$ cannot be defined as being dual to $\partial / \partial \theta^{\alpha}$, since there is no such tangent vector. Rather, $d \theta^{\alpha}$ is defined as the cotangent vector which gives $v_{l} \delta^{\alpha \beta}$ when evaluated on the tangent vector $v_{i} \partial / \partial \theta^{\beta}$.) The differential operator here will be written temporarily as $D$ and is given by

$$
D=d z^{A} \frac{\partial}{\partial z^{A}} .
$$

The cohomology is denoted $H_{G}(M)$.

The de Rham cohomology is known to have topological significance by virtue of the theorems which relate it to homology theory, which directly detects the existence of closed but nonbounding submanifolds. How are the other cohomology theories related to that of de Rham?

The relation between $H_{D R}\left(M ; B_{L}\right)$ and $H_{D R}(M)$ is quite simple. When a $B_{L}$-valued differential form is evaluated on a set of tangent vectors, the result is an element $A$ of $B_{L}$ which can be written

$$
A=A_{0}+A_{i} v_{i}+A_{i j} v_{i} v_{j}+\cdots,
$$

with real coefficients. Therefore a $B_{L}$-valued form is equivalent to $2^{L}$ real-valued forms. Furthermore, it is closed or exact precisely when each of the real-valued forms is. Therefore $H_{D R}^{k}\left(M ; B_{L}\right)$ consists of $2^{L}$ copies of $H_{D R}^{k}(M)$ and contains exactly the same information.

The relation between $H_{G}(M)$ and $H_{D R}\left(M ; B_{L}\right)$ is less clear. Both the forms and the 
derivative operators seem different. However, any $G^{\infty}$ form can be regarded as a $B_{L^{-}}$ valued form by replacing each $d z^{A}$ by $v_{\Gamma} d z_{\Gamma}^{A}$. With this correspondence, the operators $d$ and $D$ in fact agree when acting on $G^{\infty}$ forms. For example, if $f$ is a $G^{\infty}$ function then

$$
d f=d z_{\Gamma}^{A} \frac{\partial f}{\partial z_{\Gamma}^{A}}=d z_{\Gamma}^{A} v_{\Gamma} \frac{\partial f}{\partial z^{A}}=d z^{A} \frac{\partial f}{\partial z^{A}}=D f .
$$

Thus any closed or exact $G^{\infty}$ form is also closed or exact as a $B_{L}$-valued form. This means there is a map $h: H_{G}(M) \rightarrow H_{D R}\left(M ; B_{L}\right)$. It simply maps the class of a closed form in $H_{G}(M)$ to the class of the corresponding form in $H_{D R}\left(M ; B_{L}\right)$. This map $h$ is a homomorphism of the cohomology rings.

The next question is whether the map $h$ is one-to-one or onto. Consider first the zeroth cohomology $H_{D R}^{0}\left(M ; B_{L}\right)$, which consists of the $B_{L}$-valued functions which are constant on each connected component of $M$. All such functions are $G^{\infty}$, so $h$ is an isomorphism on $H^{0}$. Next consider $H^{1}$. In this case, $h$ is one-to-one but not generally onto. This statement is proven by showing that if $\omega$ is a $G^{\infty} 1$-form with $\omega=d \psi$, then $\psi$ is $G^{\infty}$. Writing out the equation $d \psi=\omega$ gives

$$
d z_{\Gamma}^{A} \frac{\partial \psi}{\partial z_{\Gamma}^{A}}=d z^{A} \omega^{A}=d z_{\Gamma}^{A} v_{\Gamma} \omega^{A}
$$

Equating coefficients,

$$
\partial \psi / \partial z_{\Gamma}^{A}=v_{\Gamma} \omega^{A},
$$

which precisely says that $\psi$ is $G^{\infty}$ and the $\omega^{A}$ are its partial derivatives.

The conclusion of this analysis is that $H_{G}^{1}(M)$ is a subgroup of $H_{D R}^{1}\left(M ; B_{L}\right)$ : the $G^{\infty}$ cohomology detects some but not all of the topology which is measured by the de Rham cohomology. For the cohomology $H^{k}, k>1$, I know of no argument that $h$ is either one-to-one or onto in general. It would be extremely interesting to know which topological properties can be detected by the $G^{\infty}$ cohomology of general supermanifolds, and what additional nontopological information it measures.

An analogous problem arises in the study of affine manifolds. There one is interested in the relation between the de Rham cohomology and the cohomology of polynomial differential forms. It is conjectured that they are isomorphic if the affine manifold is complete, and this can be proven in special cases [14,15], but little else seems to be known. The analogy between this and the $G^{\infty}$ problem rests on the fact that $G^{\infty}$ forms are polynomial in the soul coordinates.

Acknowledgement. I thank Louis Crane for helpful discussions.

\section{References}

1. Berezin, F. A.: The method of second quantization. New York: Academic Press 1966

2. Rogers, A.: Integration on supermanifolds. In: Mathematical aspects of superspace. Seifert, H.-J., Clarke, C.J.S., Rosenblum, A. (ed.) Boston: D. Reidel 1984

3. DeWitt, B. S.: Supermanifolds. Cambridge: Cambridge University Press 1984

4. Rabin, J. M.: The Berezin integral as a contour integral. In: Supersymmetry in physics. Kostelecky, 
V. A., Campbell, D. K. (ed.) Amsterdam: North-Holland, 1985. Also published as Physica 15D, 6570 (1985)

5. Rogers, A.: A global theory of supermanifolds. J. Math. Phys. 21, 1352-1365 (1980)

6. Rabin, J. M., Crane, L.: Global properties of supermanifolds. Commun. Math. Phys. 100, 141-160 (1985)

7. Rabin, J. M., Crane, L.: How different are the supermanifolds of Rogers and DeWitt? University of Chicago preprint EFI 85-25, Commun. Math. Phys. (in press)

8. Boyer, C. P., Gitler, S.: The theory of $G^{\infty}$ supermanifolds. Trans. Am. Math. Soc. 285, 241-267 (1984)

9. Berezin, F. A.: Differential forms on supermanifolds. Sov. J. Nucl. Phys. 30, 605-609 (1979)

10. Hoyos, J., Quíros, M., Ramírez Mittelbrun, J., de Urries, F. J.: Generalized supermanifolds III. $\rho$ supermanifolds. J. Math. Phys. 25, 847-854 (1984)

11. Rothstein, M.: On the resolution of some difficulties in the theory of $G^{\infty}$ manifolds. University of Washington Mathematics Department preprint 1985

12. Rogers, A.: Consistent superspace integration. J. Math. Phys. 26, 385-392 (1985)

13. Rogers, A.: On the existence of global integral forms on supermanifolds. J. Math. Phys. 26, 2749-2753 (1985)

14. Goldman, W. M., Hirsch, M. W.: Polynomial forms on affine manifolds. Pac. J. Math. 101, 115-121 (1982)

15. Goldman, W. M.: On the polynomial cohomology of affine manifolds. Invent. Math. 65, 453-457 (1982)

Communicated by S.-T. Yau

Received June 10, 1985 
